\title{
Generation of Intelligent Reports for Ubiquitous Group Decision Support Systems
}

\author{
Luís Conceição*, João Carneiro*†, Diogo Martinho*, Goreti Marreiros* and Paulo Novais ${ }^{\dagger}$ \\ *GECAD - Research Group on Intelligent Engineering and Computing for Advanced Innovation and Development, \\ Polytechnic of Porto, Porto 4200-072, Portugal \\ $\{1071223$, jomrc, diepm, mgt $\} @$ isep.ipp.pt \\ ${ }^{\dagger}$ ALGORITMI Centre, University of Minho, Guimarães 4800-058, Portugal \\ \{pjon\}@di.uminho.pt
}

\begin{abstract}
Supporting group decision-making is a complex process, especially when decision-makers have no opportunity to gather at the same place and at the same time. Besides that, finding solutions may be difficult in case agents representing decision-makers are not able to understand the process and support them accordingly. In this work we present some topics of information that can be reported to decision-makers to improve their perception about the negotiation process. We classified those topics according to two dimensions and we defined an algorithm to select which information will be built for each report.

Index Terms-Intelligent Reports; Ubiquitous Group Decision Support Systems; Decision-Making; Multi-Agent Systems.
\end{abstract}

\section{INTRODUCTION}

The decision-making process done in organizations has evolved over the last years and decisions usually made only by one person started being made by a group of people [1]. Nowadays, the success of an organization is strongly related with the quality of each decision made. Throughout the last years and with the goal to maximize the quality of decisions, several systems have been developed that aim to support decision-makers during the decision-making process.

With the evolution of technology, Group Decision Support Systems (GDSS) have also evolved and we are now facing a new paradigm in group-decision making, where we see and talk more and more about Ubiquitous GDSS. These systems first appeared with the purpose to support decision-makers with tight schedules and that are frequently travelling. If different time zones are considered it is even more difficult for these decision-makers to participate in the decision-making process at the same time. Ubiquitous GDSS intend to support the user in the group decision-making process, at any time, from anywhere, and through all kind of electronic devices [2][4].

The quality, the quantity and utility of the information presented by a GDSS to a decision-maker are key factors for the decision-making process. Looking at literature related with GDSS or even DSS in general, it is possible to find some works which propose: architectures, problem-specific models, frameworks, and other components [5]-[9]. Besides this, it is also possible to learn which information should be presented to each decision-maker and through which kind of format

978-1-5090-3608-0/16/\$31.00 (c) 2016 IEEE
[10]. However, if existing approaches make sense in GDSS of the type face-to-face, in the case of Ubiquitous GDSS, these same approaches do not make sense. In this type of context, especially when it is being considered systems that use agents to represent decision-makers, it becomes necessary to develop a tool that allows each decision-maker to obtain all relevant information about the decision-making process. This process is continuous and requires several iterations of discussion between participants. It is also necessary to provide the system with the ability to interact with decision-makers by reporting data and stimulating their participation throughout the process. This interaction is essential for the success of the application. Therefore, it is important to analyse how and which solution should result from all interactions between decision-makers and the Ubiquitous GDSS. For this, and according to the context of this work, it makes sense to think about Intelligent Reports (IR).

In this work we have identified topics of information which we assume that can be extracted from a group decisionmaking process. We classified these topics according to two dimensions and we defined an algorithm to select information to build each report.

The rest of the paper is organized as follows: in the section II we present our methods, in the section III we present the results of our case study. The following section (IV) we discuss the results that were obtained and in section $\mathrm{V}$ some conclusions are presented.

\section{MethodS}

Following our most recent work on Intelligent Reports [11], an IR should be built based on three key factors related to each decision-maker: Expertise Level, Time and Interest. The combination of these three factors results in the level of detail and complexity of the information that is presented to each decision-maker. In this work we considered that the topics identified are perceptible to any expertise level as they are only related with the group-decision making process and are not related with specific (technical) details towards alternatives or criteria. Therefore, the model proposed in this work does not consider this dimension. The information that should be included in reports is based in the information inserted by each participant through the problem configuration interface 
as presented in [3] and is also based in the information retrieved from the agents' negotiation process [12]. Intelligent Reports are elaborated with information that is relevant for each targeted decision-maker. This model should be applied to a Ubiquitous Group Decision Support System (UbiGDSS) using a group of agents to represent preferences of each real decision-maker and that way aim to reach a consensus with one or more solutions for a certain multi-criteria problem.

Defining base model to report information to decisionmakers participating in the process is a complex task due to the large amount of information that is available. It is intended that the generated report can clarify the targeted decisionmaker and show him what is happening in the decision-making process, adapting the content with factors mentioned above. The first step is to identify all topics that can be extracted from the decision-making process and then define a way to present information retrieved directly or indirectly from this process. We identified the topics bellow (information that can be presented in na UbiGDSS as referred above):

1) Alternatives supported by each decision-maker;

2) Criteria hierarchy (criteria which is considered to be the most important by decision-makers);

3) Alternative which may not be the "most preferred" by the group, but still obtain the highest level of satisfaction in for that group in case it is chosen;

4) Alternatives classification according to an analytical algorithm only considering criteria classification;

5) Analytical preference coherence by the decision-maker towards alternatives, according to the configuration and importance given to each criterion;

6) Decision-maker's satisfaction forecast towards possible solutions;

7) Reasons why a representing agent changed an alternative preference and display the reasons behind that change;

8) Other decision-makers' satisfaction forecast towards possible solutions;

9) Alternatives' preference given by credible decisionmakers;

10) Criteria preference given by credible decision-makers;

11) Information related to new preferences of other decisionmakers (accepted requests);

12) Reasons which led other decision-makers to support new alternatives;

13) Statistical data about each decision-maker's participation in the process;

14) Identify groups of decision-makers with preferences towards a certain alternative;

15) Identify groups of decision-makers with preferences towards a certain criterion;

16) Reasons (arguments) sustaining the support towards a certain alternative;

17) Reasons (arguments) sustaining the importance given to a certain criterion;

18) Information related with the proximity towards each decision-maker;
19) Forecast related with the proximity network with each other decision-maker.

In order to define which topics are relevant in each context, we think that it is important to classify each one of them according to their level of detail. For that we defined 4 levels of detail: General, General-Detailed, Specific and Very-Specific.

1) General: The level of detail is general when the information is related to problem basic questions: alternatives and criteria preferences, as well as crucial information to the perception of the state of the decision.

2) General-Detailed: This level includes more detailed topics compared to the previous one. However, these topics are still related with direct questions about the problem, such as: alternatives and criteria. Some of this information already requires some sort of processing and then presents processed or modified data.

3) Specific: This level is related with questions requiring more complex algorithms or using data generated in the decision process to create new information.

4) Very-Specific: This is the highest level of detail that is considered. This classification includes information related with artificial intelligence techniques such as forecasts or simulations of decision scenarios.

The levels of detail present a value, as seen in table I:

TABLE I

VALORIZATION OF LEVEL OF DETAIL

\begin{tabular}{l|c}
\hline Level of Detail & Value \\
\hline General & 1 \\
General-Detailed & 2 \\
Specific & 3 \\
Very-Specific & 4 \\
\hline
\end{tabular}

Besides classifying each topic according to the level of detail, we have also classified them based on the level of interest according to two dimensions (self - relevant information about the decision-maker himself; others - relevant information about the group). This evaluation has been done according to Table II:

TABLE II

VALORIZATION OF INTEREST LEVELS

\begin{tabular}{l|c}
\hline Level of Interest & Value \\
\hline High & 1 \\
Medium & 2 \\
Low & 3 \\
\hline
\end{tabular}

The interest level is related with the decision-maker behaviour style. For this proposal we have followed our previous studies [13] where we identified 5 conflict styles: Dominating, Integrating, Compromising, Obliging and Avoiding. These styles differentiate from each other by what we think that are 4 essential dimensions for this context:

- Concern for Self - This dimension is related to the individual's concern for his own opinion above the others since he is likely to adapt a more one-sided attitude dur- 
ing the decision-making process by making statements, questions and requests that detail that opinion;

- Concern for others - This dimension relates to the individual's concern for other individuals' opinion. He adapts a more altruist attitude during the decision-making process, trying to understand other opinions and making an effort to reach a decision that benefits or pleases most of the participants;

- Activity - This dimension relates to the effort put into the decision-making process by the individual, meaning that if an individual is more active he is likely to make more questions and statements and requests;

- Resistance to change - This dimension relates with the difficulty for an individual to accept other individual's opinions.

TABLE III

CONFLICT STYLES AND CORRESPONDING DIMENSIONS, ADAPTED FROM [13]

\begin{tabular}{lllll}
\hline $\begin{array}{l}\text { Behaviour } \\
\text { Style }\end{array}$ & $\begin{array}{l}\text { Concern } \\
\text { for Self }\end{array}$ & $\begin{array}{l}\text { Concern } \\
\text { for } \\
\text { Others }\end{array}$ & Activity & $\begin{array}{l}\text { Resistance } \\
\text { to } \\
\text { Change }\end{array}$ \\
\hline Dominating & 3 & 1 & 3 & 3 \\
Integrating & 3 & 3 & 3 & 3 \\
Compromising & 2 & 2 & 2 & 2 \\
Obliging & 1 & 3 & 1 & 1 \\
Avoiding & 1 & 1 & 1 & 1 \\
\hline
\end{tabular}

In Table III, we describe each conflict style by providing a value for every dimension mentioned above. The numeric values given to each dimension are correlated with the definitions established by Rahim and Magner (1995) (to learn more about this correlation see [13], [14]).

To build a report we only use the dimensions of Concern for Self and Concern for Others, since these are the dimensions that are related to the interests of decision-makers throughout the group decision-making process. These dimensions are used by our algorithm (1) to select (or not), a topic to be included in a report. From our point of view, an IR is divided in 2 parts which we name as sections. These sections are based on the topics of information. Their content is defined according to the interests of the decision-maker. The first section presents global information about the level of consensus (preference given by each decision-maker) towards each alternative. The specificity and detail given to the presentation this information has to be adjusted to the targeted decision-maker. This section is similar for each report generated which means it will present the same information regardless the targeted decision-maker. This happens since even if that decision-maker has limited availability and/or does not have any interest about the ongoing decision-making process (Avoiding), this information is the minimum information that must be included in the report. Fig. 1 and Fig. 2 are examples of this information. This way, it is possible to display the current state of the ongoing decisionmaking process.

The second section presents information about the self and other group elements. In this section it is considered information with a higher level of complexity such as forecasts

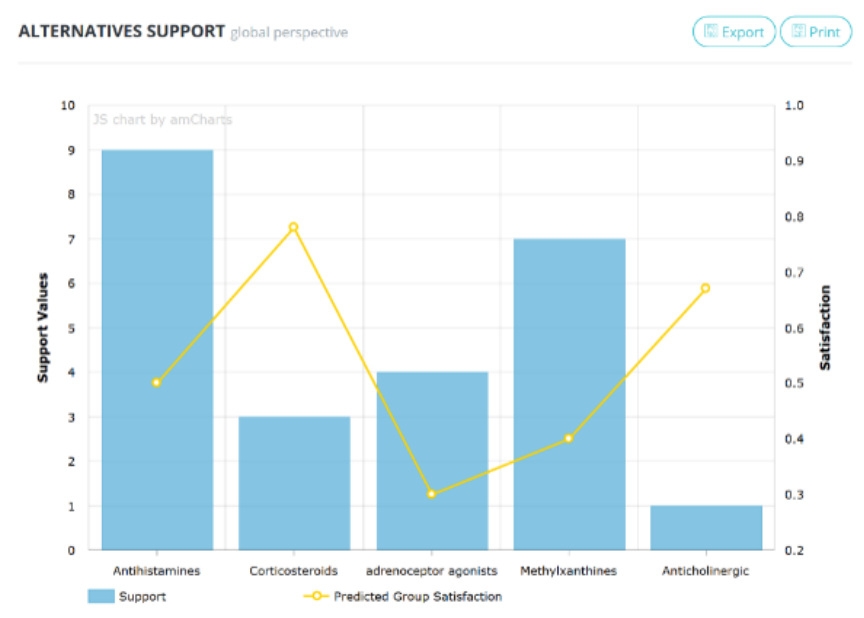

Fig. 1. Graphic with alternatives support

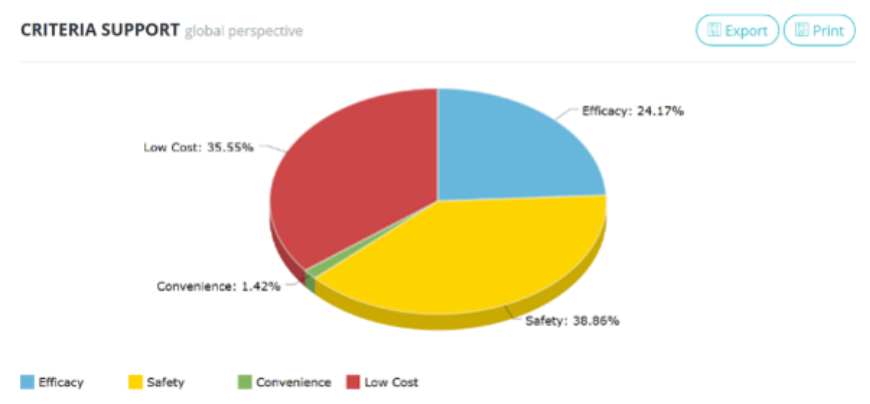

Fig. 2. Graphic with criteria support

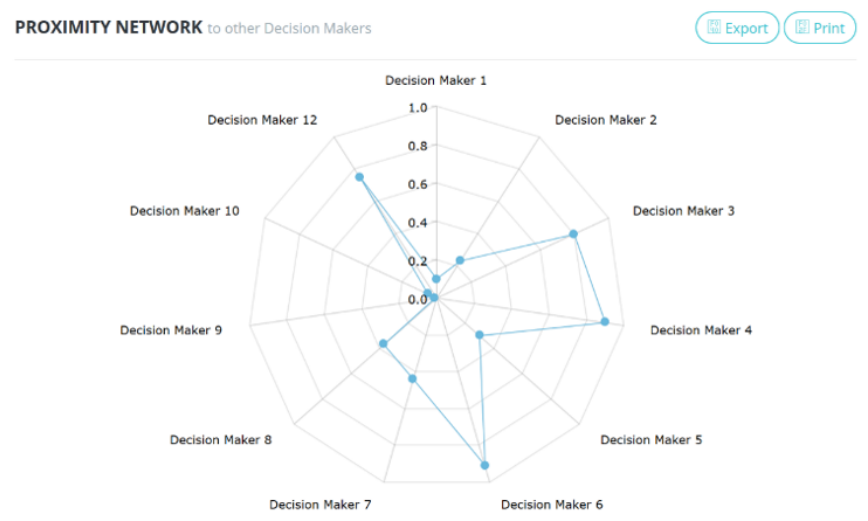

Fig. 3. Proximity Network to other decision-makers

or simulations of different scenarios, identification of different groups (concerning their preferences), as well as explanations about decisions done by the system. For example, we can measure the proximity between the decision-maker and the remaining group elements (Fig.3), or alternatives evaluation according to preferences given for each criterion, allowing the decision-maker to analyse the coherence of his choices at the moment he performed the problem configuration (Fig.4). 


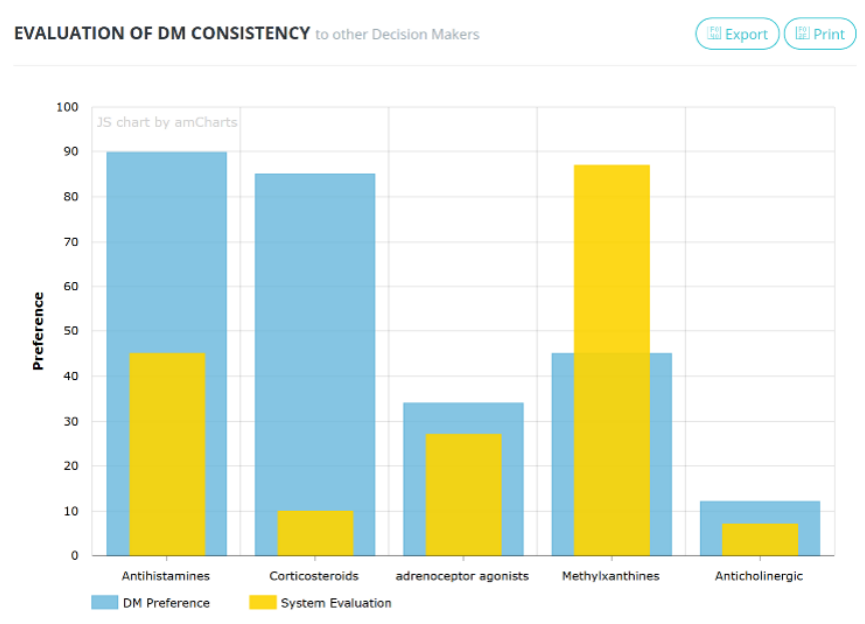

Fig. 4. Evaluation of decision-maker consistency

\section{Let topic be the topic;}

Let lTopics be the list of all topics;

Let $\mathrm{dm}$ be decision-maker;

Let $\mathrm{CO}$ be the decision-maker value for conflict for others;

Let CS be the decision-maker value for conflict for self;

Let lShowTopics be the list of topics to include in report; begin

foreach topic $\in$ lTopics do

if topic.Detail $<=d m$.Availability then

if topic.InterestForSelf $<=d m . C S$ then

lShowTopics.add(topic);

end if

else

if topic.InterestForOthers $<=d m . C O$

then

lShowTopics.add(topic);

end if

end if

end foreach

end

Algorithm 1: Selection of topics to include in reports

\section{Evaluation AND Results}

In order to validate our proposal, we ran a case study composed by a survey which was completed by a random set of people. The only constraint was that an individual completing it had to be accustomed with the decision-making process: either has the necessity to make decisions on a daily basis in their work or possesses knowledge in the area of group decision-making. The survey was divided in three distinct sections. In the first phase, every individual was requested to fill demographic data about himself/herself such as: gender, age, level of education and profession. In the next phase, each individual had to classify each one of the 19 topics according to its level of detail (General, General Specific, Specific and

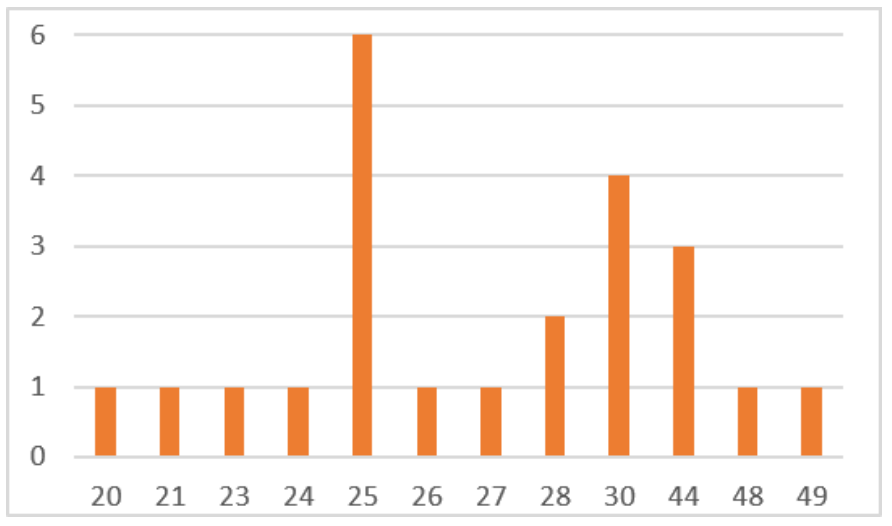

Fig. 5. Age distribution of the survey respondents

TABLE IV

PROFESSIONS OF THE SURVEY RESPONDENTS

\begin{tabular}{lll}
\hline Profession & Freq. & Relative Freq. \\
\hline Project Manager & 1 & $4 \%$ \\
Researcher & 7 & $30 \%$ \\
Computer engineer & 3 & $13 \%$ \\
Food engineer & 1 & $4 \%$ \\
Manager & 1 & $4 \%$ \\
Student & 6 & $26 \%$ \\
Consultant & 1 & $4 \%$ \\
Biologist & 1 & $4 \%$ \\
Pharmaceutical & 1 & $4 \%$ \\
Chef & 1 & $4 \%$ \\
\hline
\end{tabular}

Very Specific) based on descriptions presented in the previous phase. In the last phase each individual had to classify once again each topic according to their level of interest in the perspective of the decision-maker and the group. The case study aims to validate and classify our proposal for each topic and be able to build reports adapted to the interest of each decision-maker.

The survey was performed through an online form, and sent to individuals with a profile related to the context of this work. In total, 23 individuals participated with ages raging from 25 and 40 years (average of 30 years old and median of 27 years old). The percentage of the number of male individuals participating in the case study was of $65 \%$. Fig. 5 presents the distribution and the age frequency of the survey respondents.

Table IV presents each profession of all 23 respondents divided in a total of 10 professions, but all related with the context of decision-making.

The second part of the survey consisted in the classification of each one of the 19 topics according to the level of detail (General, General Specific, Specific and Very Specific). Table $\mathrm{V}$ and Fig. 6 show the frequencies obtained for each topic.

To obtain classifications for each topic, every individual evaluated the same topic twice: one evaluation related with the interest towards a certain information for him/her, and another evaluation with the interest for the group. Table VI and Fig. 7 present the frequencies obtained for each topic. 
TABLE V

FREQUENCIES FOR LEVEL OF DETAIL

\begin{tabular}{lllllllll}
\hline & General & \multicolumn{2}{l}{ General Detailed } & \multicolumn{2}{l}{ Specific } & \multicolumn{2}{l}{ Very Specific } \\
\hline 1 & 23 & $100 \%$ & 0 & $0 \%$ & 0 & $0 \%$ & 0 & $0 \%$ \\
2 & 17 & $74 \%$ & 6 & $26 \%$ & 0 & $0 \%$ & 0 & $0 \%$ \\
3 & 0 & $0 \%$ & 4 & $17 \%$ & 18 & $78 \%$ & 1 & $4 \%$ \\
4 & 0 & $0 \%$ & 6 & $26 \%$ & 14 & $61 \%$ & 3 & $13 \%$ \\
5 & 0 & $0 \%$ & 12 & $52 \%$ & 2 & $9 \%$ & 9 & $39 \%$ \\
6 & 0 & $0 \%$ & 2 & $9 \%$ & 10 & $43 \%$ & 11 & $48 \%$ \\
7 & 2 & $9 \%$ & 0 & $0 \%$ & 15 & $65 \%$ & 6 & $26 \%$ \\
8 & 0 & $0 \%$ & 2 & $9 \%$ & 14 & $61 \%$ & 7 & $30 \%$ \\
9 & 2 & $9 \%$ & 13 & $57 \%$ & 6 & $26 \%$ & 2 & $9 \%$ \\
10 & 0 & $0 \%$ & 8 & $35 \%$ & 11 & $48 \%$ & 4 & $17 \%$ \\
11 & 2 & $9 \%$ & 7 & $30 \%$ & 14 & $61 \%$ & 0 & $0 \%$ \\
12 & 0 & $0 \%$ & 5 & $22 \%$ & 16 & $70 \%$ & 2 & $9 \%$ \\
13 & 2 & $9 \%$ & 5 & $22 \%$ & 12 & $52 \%$ & 4 & $17 \%$ \\
14 & 0 & $0 \%$ & 8 & $35 \%$ & 9 & $39 \%$ & 6 & $26 \%$ \\
15 & 0 & $0 \%$ & 4 & $17 \%$ & 9 & $39 \%$ & 10 & $43 \%$ \\
16 & 0 & $0 \%$ & 5 & $22 \%$ & 14 & $61 \%$ & 4 & $17 \%$ \\
17 & 0 & $0 \%$ & 5 & $22 \%$ & 10 & $43 \%$ & 8 & $35 \%$ \\
18 & 0 & $0 \%$ & 1 & $4 \%$ & 2 & $9 \%$ & 20 & $87 \%$ \\
19 & 0 & $0 \%$ & 0 & $0 \%$ & 1 & $4 \%$ & 22 & $96 \%$ \\
\hline
\end{tabular}

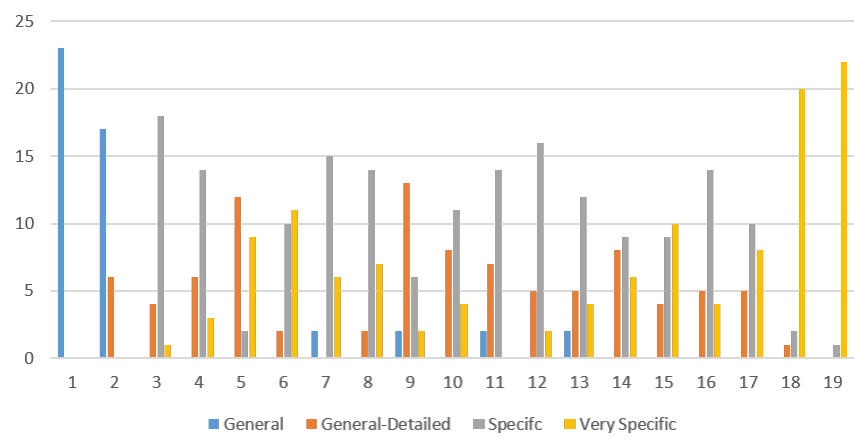

Fig. 6. Level of detail results

\section{DISCUSSION}

As mentioned above there are not existing works in literature related with reporting information in GDSS. The goal of this work is to make it easier to adopt and use group decision support systems inside organizations. Although this issue is not explored in this work it known that GDSS are not yet accepted by organizations for several reasons, with one being the lack of trust that each decision-maker has in the system. Creating intelligent reports will contribute to strengthen that trust since it is intended to show and explain to each decision-maker what is really happening during a group decision-making process. In this work we only intend to identify what information is considered relevant for each decision-maker when creating intelligent reports.

Looking at the results obtained towards the classification of the level of detail for each topic, we verified that the General detail has two topics ( 1 and 2 ) with the first being classified as General by $100 \%$ of the respondents while the second was classified by $74 \%$. The General Detailed has the topics 5 and 9 with the most number of votes. The Specific category has the topics $3,4,7,8$, and 10 to 17 with most number of votes. The Very Specific category includes topics 6, 18 and 19.
TABLE VI

FREQUENCIES OF LEVEL OF INTEREST

\begin{tabular}{lllllll} 
& Low & \multicolumn{3}{l}{ Medium } \\
\hline 1.Others & 2 & $9 \%$ & 7 & $30 \%$ & 14 & $61 \%$ \\
2.Self & 2 & $9 \%$ & 4 & $17 \%$ & 17 & $74 \%$ \\
2.Others & 2 & $9 \%$ & 8 & $35 \%$ & 13 & $57 \%$ \\
3.Self & 0 & $0 \%$ & 9 & $39 \%$ & 14 & $61 \%$ \\
3.Others & 2 & $9 \%$ & 16 & $70 \%$ & 5 & $22 \%$ \\
4.Self & 0 & $0 \%$ & 8 & $35 \%$ & 15 & $65 \%$ \\
4.Others & 0 & $0 \%$ & 15 & $65 \%$ & 8 & $35 \%$ \\
5.Self & 5 & $22 \%$ & 5 & $22 \%$ & 13 & $57 \%$ \\
5.Others & 4 & $17 \%$ & 12 & $52 \%$ & 7 & $30 \%$ \\
6.Self & 7 & $30 \%$ & 8 & $35 \%$ & 8 & $35 \%$ \\
6.Others & 2 & $9 \%$ & 11 & $48 \%$ & 10 & $43 \%$ \\
7.Self & 11 & $48 \%$ & 5 & $22 \%$ & 7 & $30 \%$ \\
7.Others & 2 & $9 \%$ & 8 & $35 \%$ & 13 & $57 \%$ \\
8.Self & 11 & $48 \%$ & 2 & $9 \%$ & 10 & $43 \%$ \\
8.Others & 0 & $0 \%$ & 15 & $65 \%$ & 8 & $35 \%$ \\
9.Self & 4 & $17 \%$ & 4 & $17 \%$ & 15 & $65 \%$ \\
9.Others & 0 & $0 \%$ & 15 & $65 \%$ & 8 & $35 \%$ \\
10.Self & 4 & $17 \%$ & 4 & $17 \%$ & 15 & $65 \%$ \\
10.Others & 0 & $0 \%$ & 13 & $57 \%$ & 10 & $43 \%$ \\
11.Self & 6 & $26 \%$ & 0 & $0 \%$ & 17 & $74 \%$ \\
11.Others & 9 & $39 \%$ & 13 & $57 \%$ & 1 & $4 \%$ \\
12.Self & 2 & $9 \%$ & 13 & $57 \%$ & 8 & $35 \%$ \\
12.Others & 7 & $30 \%$ & 15 & $65 \%$ & 1 & $4 \%$ \\
13.Self & 2 & $9 \%$ & 14 & $61 \%$ & 7 & $30 \%$ \\
13.Others & 13 & $57 \%$ & 6 & $26 \%$ & 4 & $17 \%$ \\
14.Self & 8 & $35 \%$ & 9 & $39 \%$ & 6 & $26 \%$ \\
14.Others & 0 & $0 \%$ & 19 & $83 \%$ & 4 & $17 \%$ \\
15.Self & 0 & $0 \%$ & 8 & $35 \%$ & 15 & $65 \%$ \\
15.Others & 2 & $9 \%$ & 18 & $78 \%$ & 3 & $13 \%$ \\
16.Self & 2 & $9 \%$ & 7 & $30 \%$ & 14 & $61 \%$ \\
16.Others & 0 & $0 \%$ & 20 & $87 \%$ & 3 & $13 \%$ \\
17.Self & 2 & $9 \%$ & 7 & $30 \%$ & 14 & $61 \%$ \\
17.Others & 2 & $9 \%$ & 20 & $87 \%$ & 1 & $4 \%$ \\
18.Self & 2 & $9 \%$ & 10 & $43 \%$ & 11 & $48 \%$ \\
18.Others & 4 & $17 \%$ & 11 & $48 \%$ & 8 & $35 \%$ \\
19.Self & 4 & $17 \%$ & 7 & $30 \%$ & 12 & $52 \%$ \\
19.Others & 7 & $30 \%$ & 7 & $30 \%$ & 9 & $39 \%$ \\
\hline & & & & & &
\end{tabular}

Although topics with the same vote percentage in more than one category it was possible to verify that, for example, in topics 14 and 15, for each considered category, the difference was just of one vote. The first case (14) obtained 8 votes in the General Detailed category and 9 in the Specific category. The second case (15) obtained 9 votes in the Specific category and 10 in the Very Specific.

Looking at the results obtained in the classification of each topic according to the level of interest it is possible to observe that $71 \%$ (15) of the topics obtained a different classification in the self and group perspective. In the decision-maker perspective, only topic 13 obtained a majority (57\%) in the Low level, while in the group perspective, topics 6 and 7 obtained that classification. Looking at the results for the Medium level, in the decision-maker perspective, topics $3,6,8$ to 12 and 14 to 18 obtained a majority. In the group perspective, topics 11 to 13 and 19 obtained the same classification. For the High level, in the decision-maker perspective, topics 1, 2, 7 and 19 obtained the majority. In the group perspective, topics 1 to 4 , 8 to 10 and 14 to 18 obtained that classification. It was also observed a tie between the classification as Medium and High for topic 5 concerning group perspective, with $35 \%$ of votes for each classification. 


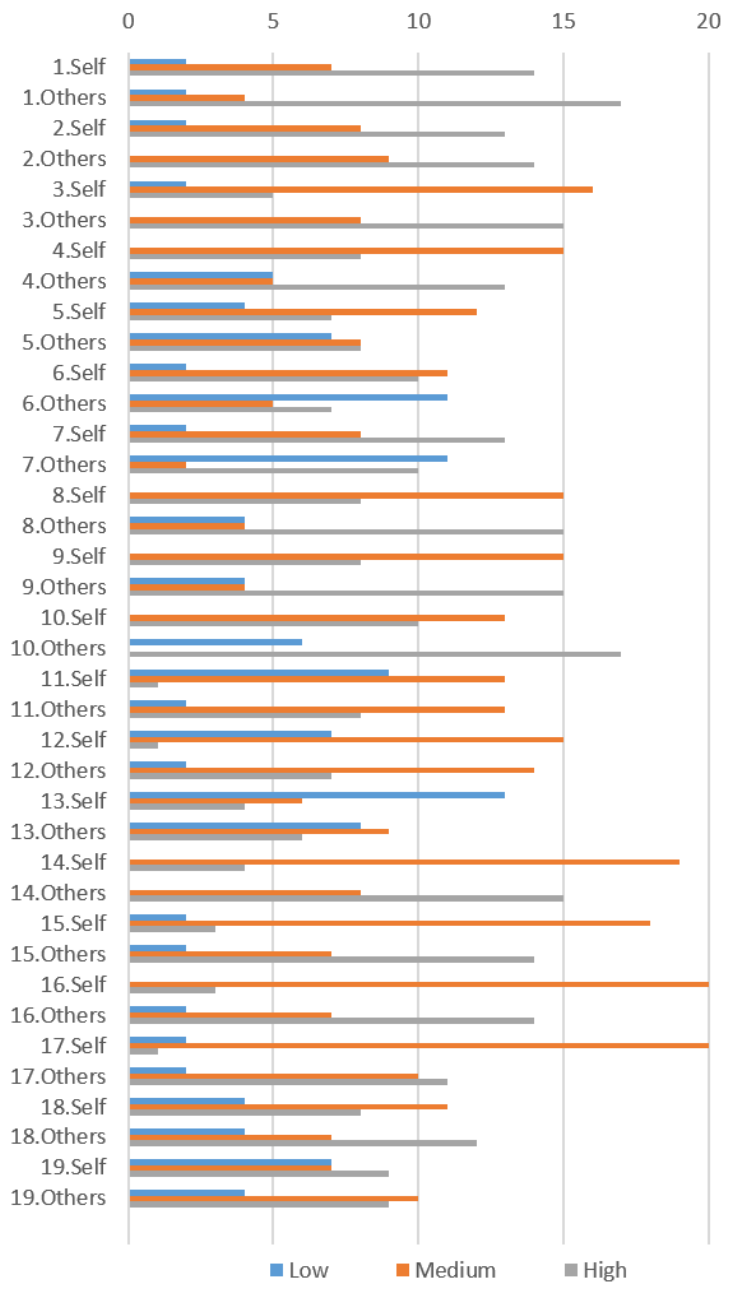

Fig. 7. Level of interest results

We think that it was possible to obtain good results with the case study, despite the survey complexity, as it was necessary to understand the concepts in order to correctly answer each question.

\section{Conclusions And Future Work}

Defining a base model to report information to decisionmakers participating in a group decision-making process is a complex task due to the quantity of available information. However, it is important to express that failing to report information to decision-makers can compromise the success of the GDSS because they will not be able to know what really happened throughout the decision-making process and understand the exposed results, and then lose trust in the system. The work here presented is the culmination of a large research work in the area of GDSS.

This work had the main goal to define the classification of different topics of information that can be used when creating an intelligent report for GDSS. Our classification proposal was structured in two phases: classifying each topic towards the level of detail and towards the level of interest in both decision-maker and group perspectives. In order to classify correctly each topic, a case study was performed where a group of decision-makers completed an online survey. We consider that the results obtained were very acceptable and satisfactory according to the goal set for this work.

The next step will be implementing the algorithm presented in this work and perform another case study using a ubiquitous GDSS. This way we want decision-makers to be able to validate reports created by the system and also the relevance of information presented in them.

\section{ACKNOWLEDGMENTS}

This work has been supported by COMPETE Programme (operational programme for competitiveness) within project POCI-01-0145-FEDER-007043, by National Funds through the FCT - Portuguese Foundation for Science and Technology within the Projects UID/CEC/00319/2013, UID/EEA/00760/2013, and the João Carneiro $\mathrm{PhD}$ grant with the reference SFRH/BD/89697/2012.

\section{REFERENCES}

[1] F. Luthans, "Organizational behavior," McGraw-Hill/Irwin, vol. 46, p. $594,2011$.

[2] G. P. Huber, "Issues in the design of group decision support systems," MIS Quarterly: Management Information Systems, vol. 8, pp. 195-204, 1984.

[3] J. Carneiro, D. Martinho, G. Marreiros, and P. Novais, "A general template to configure multi-criteria problems in ubiquitous gdss," International Journal of Software Engineering and Its Applications, vol. 9, pp. 193-206, 2015.

[4] M. Weiser, "Ubiquitous computing," Computer, vol. 26, pp. 71-72, 1993.

[5] D.-Y. Choi, "Aggregation of preferences based on fsam in gdss," Systems, Man and Cybernetics, Part A: Systems and Humans, IEEE Transactions on, vol. 38, no. 1, pp. 2-8, 2008.

[6] M. Miranda, A. Abelha, M. Santos, J. Machado, and J. Neves, A group decision support system for staging of cancer. Springer, 2008, pp. 114-121.

[7] G. Marreiros, R. Santos, C. Ramos, and J. Neves, "Context-aware emotion-based model for group decision making," Intelligent Systems, IEEE, vol. 25, pp. 31-39, 2010.

[8] M. Tavana, M. Behzadian, M. Pirdashti, and H. Pirdashti, "A prometheegdss for oil and gas pipeline planning in the caspian sea basin," Energy Economics, vol. 36, pp. 716-728, 2013.

[9] R. V. Efremov and A. V. Lotov, "Multi-criteria remote asynchronous group decision screening: an experimental study," Group Decision and Negotiation, vol. 23, no. 1, pp. 31-48, 2014.

[10] G. L. Brase, "Which statistical formats facilitate what decisions? the perception and influence of different statistical information formats," Journal of Behavioral Decision Making, vol. 15, no. 5, pp. 381-401, 2002.

[11] J. Carneiro, L. Conceição, D. Martinho, G. Marreiros, and P. Novais, "Intelligent reports for group decision support systems," in Workshop Proceedings of the 12th International Conference on Intelligent Environments, 2016, Conference Proceedings.

[12] J. Carneiro, D. Martinho, G. Marreiros, and P. Novais, "Intelligent negotiation model for ubiquitous group decision scenarios," Frontiers of Information Technology \& Electronic Engineering, vol. 17, no. 4, pp. 296-308, 2016.

[13] D. Martinho, J. Carneiro, G. Marreiros, and P. Novais, "Dealing with agents' behaviour in the decision-making process," in Workshop Proceedings of the 11th International Conference on Intelligent Environments, vol. 19. IOS Press, 2015, Conference Proceedings, p. 4.

[14] M. A. Rahim and N. R. Magner, "Confirmatory factor analysis of the styles of handling interpersonal conflict: first-order factor model and its invariance across groups," Journal of applied psychology, vol. 80, no. 1, p. 122,1995 . 